\title{
Molecular markers for plant breeding: comparisons of RFLP and RAPD genotyping costs
}

\author{
M. Ragot ${ }^{1}$, D. A. Hoisington ${ }^{2}$ \\ ${ }^{1}$ Department of Genetics, North Carolina State University, Raleigh, NC 27695-7614, USA \\ 2 Applied Molecular Genetics Laboratory, CIMMYT, Apdo. Postal 6-641, 06600 Mexico DF, Mexico
}

Received: 18 may 1992 / Accepted: 28 January 1993

\begin{abstract}
Three molecular marker protocols, chemiluminescent restriction fragment length polymorphisms (c-RFLPs), radioactivity-based restriction fragment length polymorphisms (r-RFLPs), and randomly amplified DNA polymorphisms (RAPDs) were compared in terms of cost and time efficiency. Estimates of cost of supplies and time requirements were obtained from simulations of maize (Zea mays L.) genotyping experiments utilizing protocols currently in use. The increase in total cost with increasing numbers of individuals genotyped and markers analyzed is higher for RAPDs than for RFLPs. RAPDs were generally found to be more cost and time efficient for studies involving small sample sizes, while RFLPs have the advantage for larger sample sizes. Because of the shorter exposure times involved, c-RFLPs require less time than $\mathrm{r}-$ RFLPs to obtain a given amount of information. Variations in the protocols, such as number of re-uses of Southern blots or cost of Taq DNA polymerase per reaction of amplification, also affect the relative merits of RAPDs and RFLPs. Two examples were analyzed where molecular markers are used: a germ plasm survey and quantitative trait loci (QTL) mapping in a segregating population. No protocol was found to be the most cost and time efficient over the entire range of sample sizes and number of marker loci studied.
\end{abstract}

Key words: Chemiluminescence - Genotyping costs RAPD - RFLP - Zea mays

Communicated by A. L. Kahler

Correspondence to: D. A. Hoisington

\section{Introduction}

Since associations between genetic markers and quantitative traits were first reported (Sax 1923), much attention has been given to the potential uses of markers in breeding programs. Such uses can generally be divided into two groups based on whether or not they require associations between markers and quantitative trait loci (QTLs) (Dudley 1989). Fingerprinting and the determination of relationships among individuals (for instance, to assign individuals to heterotic groups) have long involved markers (Bassiri and Adams 1978; Goodman and Stuber 1980; Soller and Beckmann 1983; Smith and Smith 1989; Smith et al. 1991) and do not require associations between markers and QTLs. The identification of favorable genes, their introgression into elite germ plasm, and selection for them in a breeding program, on the other hand, require linkages between markers and QTLs (Stuber and Edwards 1986; Osborn et al. 1987; Keim et al. 1990).

Not all types of markers are suitable for breeding applications. Morphological and cytological markers are typically associated with deleterious effects or are difficult to analyze in breeding populations and thus are of very little use (Tanksley et al. 1989). Molecular markers, in contrast, are generally free of such associations. Extensive use has been made of isozyme markers to characterize genetic diversity (Bassiri and Adams 1978; Doebley et al. 1984; Smith et al. 1985a, b; Kesseli and Michelmore 1986; McIntyre 1988; Asins and Carbonell 1989). The paucity of isozyme loci and their associated polymorphisms, however, restricts their usefulness in breeding (Helentjaris et al. 1986; Dudley 1989; Tanskley et al. 1989). DNA polymorphisms such as restriction fragment length polymorphisms (RFLPś) (Grodzicker et al. 1974) and randomly 
amplified DNA polymorphisms (RAPDs) (Welsh and McClelland 1990; Williams et al. 1990) have overcome these limitations in many species. Both types of markers have been used for the construction of genetic maps in a variety of species including maize (Helentjaris et al. 1986; Hoisington and Coe 1990), barley (Shin et al. 1990; Heun et al. 1991; Graner et al. 1991), soybean (Keim et al. 1990), rice (McCoucgh et al. 1988), tomato (Helentjaris et al. 1986), potato (Gebhardt et al. 1989; 1991), Brassica rapa (Song et al. 1991), lettuce (Landry et al. 1987), loblolly pine (Devey et al. 1991), and Arabidopsis (Chang et al. 1988; Nam et al. 1989), and are very promising for breeding applications (Beckmann and Soller 1983; Murray et al. 1988; Tanksley et al. 1989; Walton 1990; Rafalski et al. 1991).

The efficiency of a breeding strategy is usually measured in terms of genetic progress per unit of time, or genetic gain (Fehr 1987). The associated cost is also of concern to most breeding programs. One of the major potential benefits of using molecular markers in breeding is the considerable savings in the time re- quired to achieve a certain amount of genetic progress (Burr et al. 1983; Dudley 1989; Tanksley et al. 1989). However, the use of markers in breeding is currently limited by the cost of and the time needed for measuring molecular marker phenotypes (De Verna and Alpert 1990).

Numerous factors affect the costs and benefits of using molecular markers for breeding (Dudley 1989), one of which is the type of marker. Reports on genotyping costs are rather scarce (Beckmann and Soller 1983; Walton 1990), and contradictory statements have been made when evaluating the relative merits of RFLPs and RAPDs in terms of cost or time (DeVerna and Alpert 1990; Martin et al. 1991; Rafalski et al. 1991; Weining and Langridge 1991; Williams et al. 1991). In this paper we compare three classes of molecular markers in terms of cost and time requirements: radioactivity-based RFLPs (r-RFLPs); non-radioactive, chemiluminescent-based RFLPs (c-RFLPs); and RAPDs. Our results are based on simulations of maize (Zea mays L.) genotyping experiments.

Table 1. Procedures and steps constituting the c-RFLP, r-RFLP and RAPD protocols

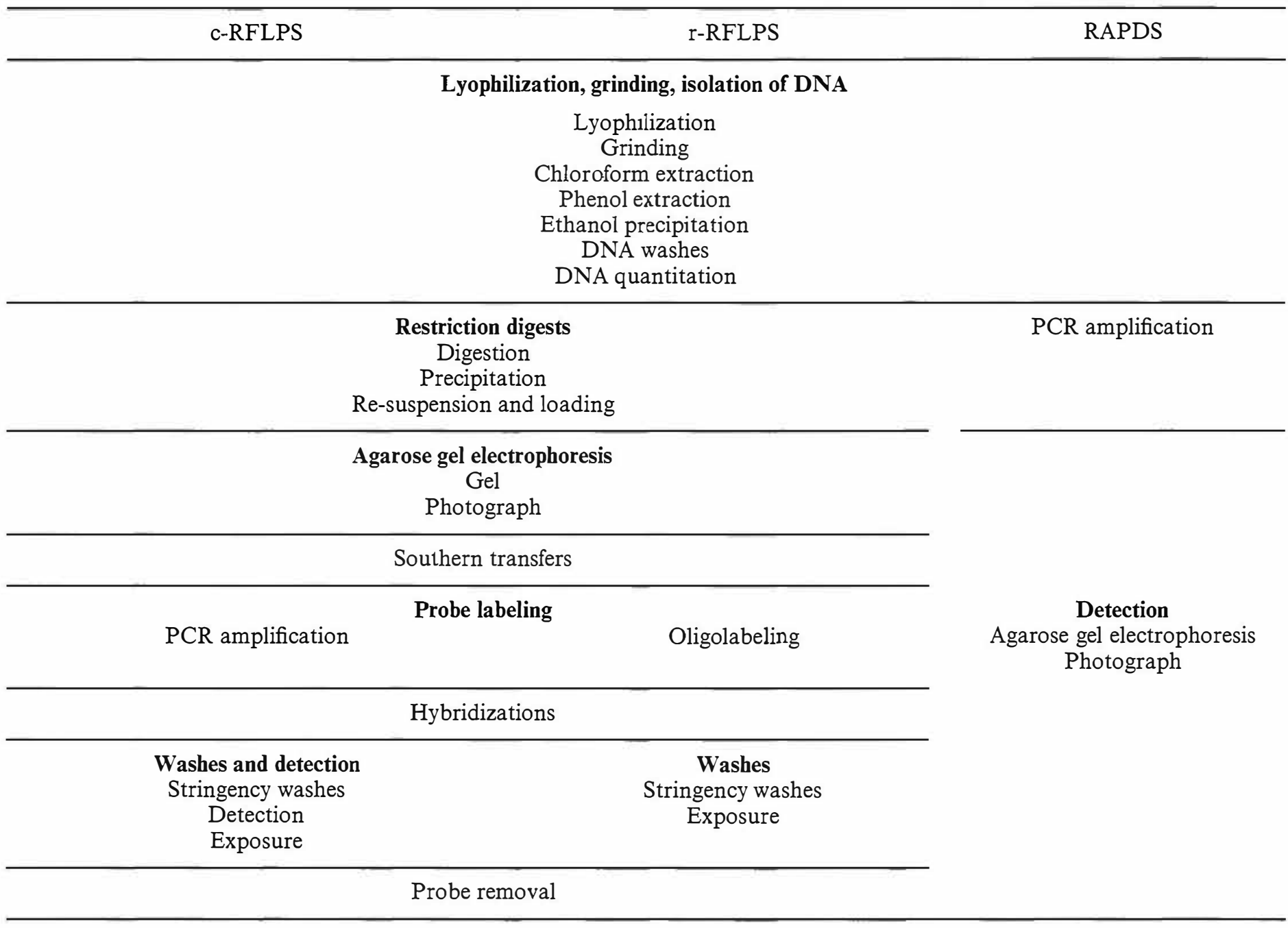




\section{Materials and methods}

\section{Genotyping protocols}

For clarity the term protocol will be used when referring to an individual genotyping technique: r-RFLPs, c-RFLPs, or RAPDs. The various parts of the protocol will be termed procedures, and the individual constituents of the procedures will be referred to as steps (Table 1). Protocols are those in use in the Applied Molecular Genetics Laboratory at CIMMYT and have been described in detail by Hoisington (1992).

\section{$r-R F L P S$}

Genomic DNA was isolated from lyophilized maize leaf or cob samples, quantified, digested using 2.5 units of restriction enzyme per microgram of DNA, and electrophoresed in agarose gels $(10 \mu \mathrm{g}$ per well). Up to $120 \mathrm{DNA}$ samples can be loaded in a single gex. Following electrophoresis, the DNA was denatured and blotted onto a nylon membrane. Probes were labeled by random priming using $50 \mu \mathrm{Ci}$ per $100 \mathrm{ng}$ of probe. Hybridizations are conducted in a hybridization oven. Up to eight membranes can be hybridized at once, in one glass bottle, with a given probe, and as many as ten probes can be used simultaneously (oven capacity is ten glass bottles). Following hybridization, the membranes underwent a series of stringency washes and were then exposed to X-ray film for the appropriate time (overnight to 5 days). Membranes were re-used after probe removal.

\section{$c-R F L P S$}

The isolation of genomic DNA, restriction digests, gel electrophoresis, and Southern transfers are performed as described above. Probes were PCR-labeled by incorporation of digoxigenin-11-dUTP (Boehringer Mannheim). Hybridizations were conducted in a hybridization oven as described for r-RFLPs. DNA-DNA hybridizations were detected with anti-digoxigenin (Boehringer Mannheim) coupled to an alkaline-phosphatasecatalyzed chemiluminescent decomposition of AMPPD $^{\circledR}$ (Tropix). Membranes were exposed overnight to X-ray film. After probe removal, membranes were re-used several times.

\section{RAPDS}

DNA isolation was carried out as previously described. Amplification reactions were performed in volumes of $25 \mu \mathrm{l}$ containing $30 \mathrm{ng}$ of genomic DNA, $30 \mathrm{ng}$ of primer and $0.01 \mathrm{mM}$ each of dATP, dCTP, dGTP, and dTTP. Various amounts of Taq DNA polymerase were used in the reactions, although cost estimates were based on the actual cost of the enzyme in the reaction. The number of amplification cycles was 40 . Amplification products were analyzed by agarose gel electrophoresis. Permanent records were obtained by photographing ethidum bromidestained gels under UV light with positive/negative Polaroid ${ }^{\circledR}$ film.

\section{Cost and time estimates}

Three calculation sheets were developed using Microsoft ${ }^{\circledR}$ Excel on an Apple Macintosh ${ }^{\circledR}$ computer to estimate genotyping costs (supplies) for each of the three protocols described above. These worksheets provide estimates of supply quantities and associated costs for any genotyping project. They are available on diskette upon request. Cost estimates are the result of simulations of genotyping projects, the characteristics of which (number of individuals, number of marker loci, etc.) were arbitrarily chosen. Amounts of supplies (reagents, plasticware) and their associated costs (in US \$) are calculated for each step of the protocol. Partial totals are computed for each procedure and summed to provide an estimate of the overall project cost. Calculations were based on $100 \%$ success for all of the laboratory work and US catalog prices from either 1991 or 1992 (where available). Estimates of cost per data point are based on the assumption that one marker (RFLP probe/restriction enzyme combination or RAPD primer) provides information at a single locus. A data point is therefore defined as the genotype of one DNA sample (one individual or a bulk of individuals) at one marker locus. It should be noted that both RFLP and RAPD analyses often provide information at more than one locus and that the use of fingerprinting probes could lead to a substantially lower cost per data point.

RFLP costs are calculated assuming that probes are available free of charge. In addition, because PCR amplification of probes allows an almost infinite supply of probes from initial plasmid inserts without further recourse to bacterial transformation, plasmid preparations, or the isolation of plasmid inserts, these latter procedures were not included in the calculations of RFLP costs. PCR amplification and the labeling of probes were taken into consideration when estimating c-RFLP costs. Characteristics of RFLP projects included restriction digests of genomic DNA with HindIII, EcoRI, and BamHI in a 2:2:1 ratio (two digestions of each DNA sample with HindIII, two with EcoRI, and one with BamHI for a total of five restriction of each DNA sample), and a maximum of five uses of each membrane (unless otherwise indicated). c-RFLP cost estimates are also based on five re-uses of the AMPPD ${ }^{\circledR}$ solution. RAPD cost estimates are based on a cost of Taq DNA polymerase per amplification reaction equal to $\$ 0.05$, unless otherwise stated.

Variation in total cost and cost per data point were calculated for several combinations of numbers of individuals and numbers of marker loci. Variations in the cost of Taq DNA polymerase per amplification (reflecting both variations in number of units used and variations in cost per unit) were studied for their effect on cost per data point of the RAPD protocol. Effects of membrane usage of RFLP genotyping costs were also investigated. Lastly, two genotyping situations were simulated: a germ plasm survey involving the genotyping of 100 individuals with 100 probes (or primers) and QTL mapping in a segregating population involving 500 progenies genotyped with 100 probes (or primers). In both cases, total cost estimates were further allocated to the various procedures of each protocol.

Estimates of actual manipulation, or "hands-on", time requirements were generated only for the genotyping of 500 individuals with 100 probes (or primers) using either c-RFLPs, r-RFLPs, or RAPDs. They do not include steps before genomic DNA isolation, nor steps beyond data acquisition (X-ray film or UV picture). It should be noted that data entry and analysis is often the most time-consuming step, especially in fingerprinting studies. Hands-on time differs from the elapsed time required to complete a step (Walton 1990). The difference between the two is primarily due to the presence of waiting periods and the ability to fit time constraints (sequence of a manipulation and waiting periods) imposed by specific steps within 1 working day.

Costs for labor were not included but would be similar for each protocol, differing only in the total cost due to the different estimates of total time for each protocol.

\section{Results}

As expected, for all three protocols total costs increased with both the number of individuals genotyped and the number of markers used. (Fig. 1a-c). The 
increase was by and large linear. Deviations from linearity that were observed for RFLPs can be attributed to the more efficient use of nylon membranes imposed by project characteristics (number of individuals and molecular markers). For small sample sizes (25 individuals), the lowest genotyping costs were obtained with RAPDs In contrast, genotyping would be performed at lowest cost for intermediate sample sizes (50-250 individuals) with c-RFLPs, and for large sample sizes (500 or more individuals) with r-RFLPs. The cost advantage of r-RFLPs over c-RFLPs for large sample sizes was found to be minimal and never exceeded $4 \%$ of the total cost. Similar results were obtained when analyzing cost per data point $\left(\mathrm{F}_{1} \mathrm{~g} 2 \mathrm{a}-\mathrm{c}\right)$ For a given sample size, cost per data point always decreased when the number of probes (primers) increased. Reduction in cost per data point was also observed when sample size increased, except for RAPDs where sample size had no measurable effect on cost per data point (data not shown). The relative merits of RFLPs and RAPDs depended mostly on the cost of Taq DNA polymerase associated with each amplification reaction (Fig 2a-c).

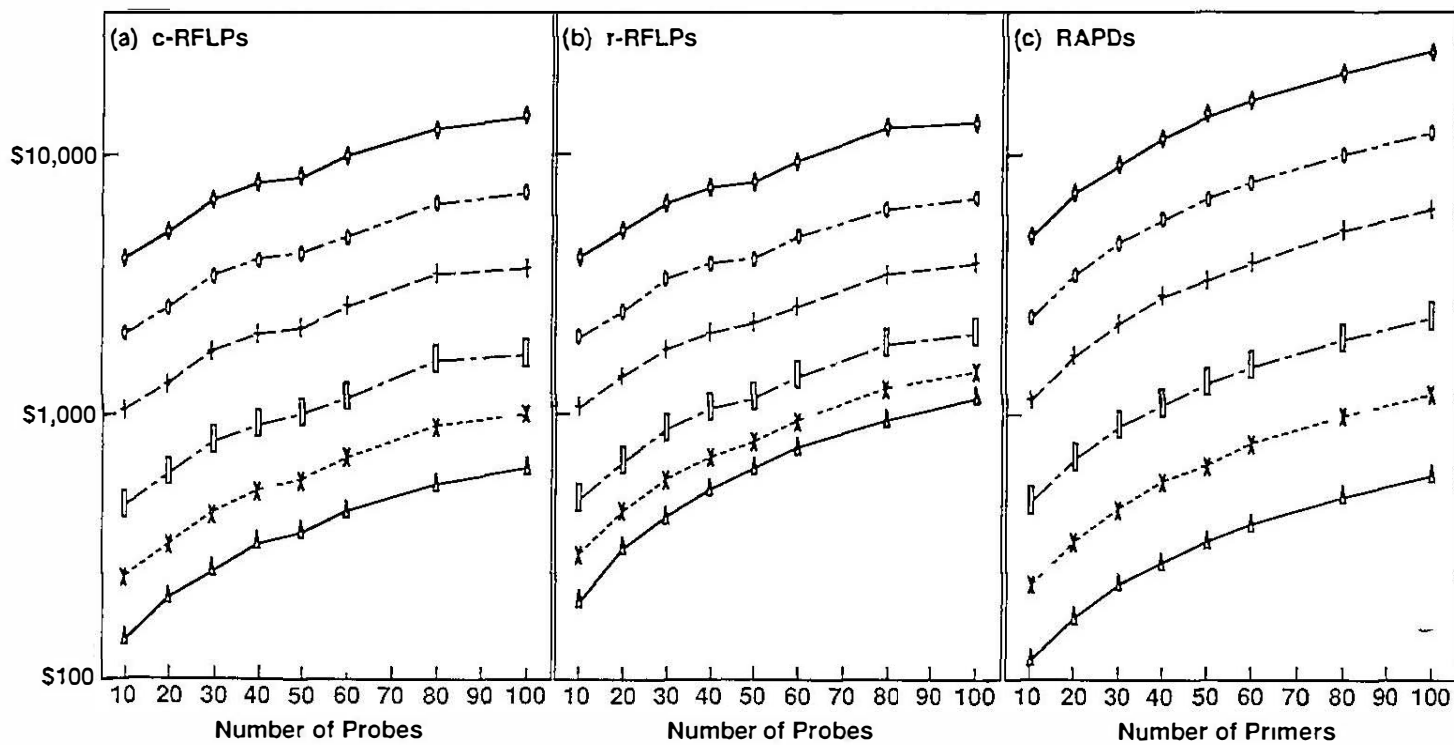

Number of Samples
$+25 \rightarrow 100 \rightarrow-500$
$-50+250 \rightarrow-1000$

Fig. 1a-c. Genotyping costs (supplies only), in relation to number of individuals and number of probes (primers), for three different molecular marker protocols a Chemiluminescent-based RFLPs (c-RFLPs), b radioactivity-based RFLPs (r-RFLPs), and c RAPDs

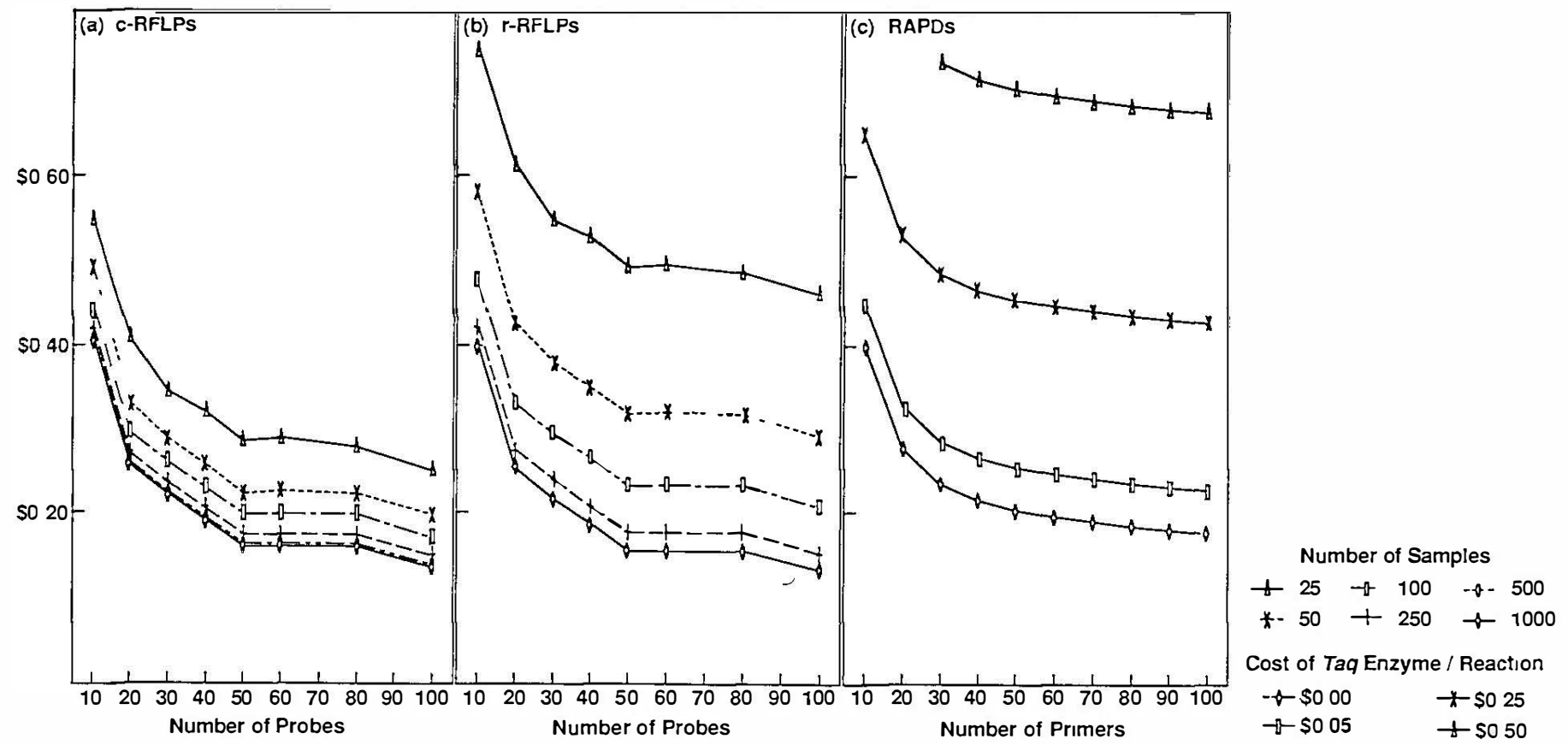

Fig. 2a-c. Cost per data point (supplies only), in relation to number of individuals and number of probes, for two different molecular marker protocols a Chemiluminescent-based RFLPs (c-RFLPs) and b radioactivity-based RFLPs (r-RFLPs) c Cost per data point (supplies only) in relation to number of primers and cost of Taq DNA polymerase per reaction of amplification for RAPDs 
Therefore, the cost per data point for RAPDs was calculated for varying costs of Taq enzyme per reaction. With a cost of Taq DNA polymerase of $\$ 0.00$ per amplification, the costs per data point for RAPDs roughly corresponded to the analysis of 50 samples using c-RFLPs and 100 samples using r-RFLPs. Therefore, if more than 50 samples were to be analyzed, c-RFLPs would result in the lowest cost per data point and RAPDs, the highest. In comparison, with a cost of Taq DNA polymerase of $\$ 0.25$ per amplification, RAPDs resulted in costs per data point equivalent to the analysis of only 25 samples using r-RFLPs and less than 25 samples using c-RFLPs.

Cost per data point ranged from $\$ 0.14$ to $\$ 0.55$ for c-RFLPs, $\$ 0.13$ to $\$ 0.76$ for r-RFLPs, and $\$ 0.18$ to $\$ 1.30$ for RAPDs (for $\$ 0.00-\$ 0.50$ cost for Taq DNA polymerase per amplification). In all cases, the lowest costs corresponded to the genotyping of 1,000 individuals with 100 probes using RFLPs or any number of individuals with 100 primers and zero cost for Taq enzyme using RAPDs. The highest costs were observed for the genotyping of 25 individuals with 10 probes using RFLPs or any number of individuals with 10 primers and $\$ 0.50$ for Taq enzyme using RAPDs. In a companion sutdy (unpublished), we estimated costs based on prices of supplies available in Mexico. While the cost per data point remained within the range given above for c-RFLPs and RAPDs, it reached as much as $\$ 1.72$ for the genotyping of 25 individuals with 10 probes for r-RFLPs. A single component, $\left[{ }^{32} \mathrm{P}\right]-$ labeled dCTP (priced in Mexico about six times as much as in the USA), is responsible for most of this increase. The cost of r-RFLPs renders the use of this protocol prohibitive in Mexico, and most likely in many other parts of the world where $\left[{ }^{32} \mathrm{P}\right]$-labeled $\mathrm{dCTP}$ is expensive.

Membrane usage greatly affected RFLP genotyping costs. Figure 3 shows the results of genotyping 500 individuals with 100 probes when membrane usage varied from one to ten re-uses. Total cost decreased by more than $75 \%$ when membrane usage rose from one to ten. More than $90 \%$ of these savings are realized between one and five uses of the membranes, due to the roughly inversely proportional relation that exists between restriction digest, gel electrophoresis and Southern transfer costs and the number of membrane uses. When membranes are used three or more times, both RFLP methods are expected to be more cost efficient than RAPDs.

To better understand variations in cost, total costs were partitioned among the procedures constituting each protocol. Changes in relative costs associated with these procedures were then monitored over a range of sample sizes and numbers of marker loci (data not shown). For a given sample size, increasing the number of probes or primers with which individuals were to be genotyped resulted in a very significant reduction in DNA extraction costs relative to other procedures. For example, for the genotyping of 100 individuals, DNA extractions would be $44 \%$ of the total cost of analyzing 10 probes (primers) and only $10 \%$ of the total cost of analyzing 100 probes (primers) (data not shown). This is a major cause for the observed decrease in cost per data point when the number of markers increased. Similarly, for a given number of RFLP probes, we observed a very large reduction in relative probe labeling costs when sample size increased. For example, for the analysis of 100 probes using c-RFLPs, probe labeling would represent $15 \%$ of the total cost for 100 individuals and only $4 \%$ of the total cost for 500 individuals (compare Fig. $4 \mathrm{a}$ and Fig. 6a). This reduction in labeling costs largely explains both the decrease in cost per data point for RFLPs and discrepancy between RFLPs and RAPDs for their variations in cost per data point when sample size increases.

Figure $4 \mathrm{a}-\mathrm{c}$ gives genotyping costs for a germ plasm survey (100 individuals genotyped with 100

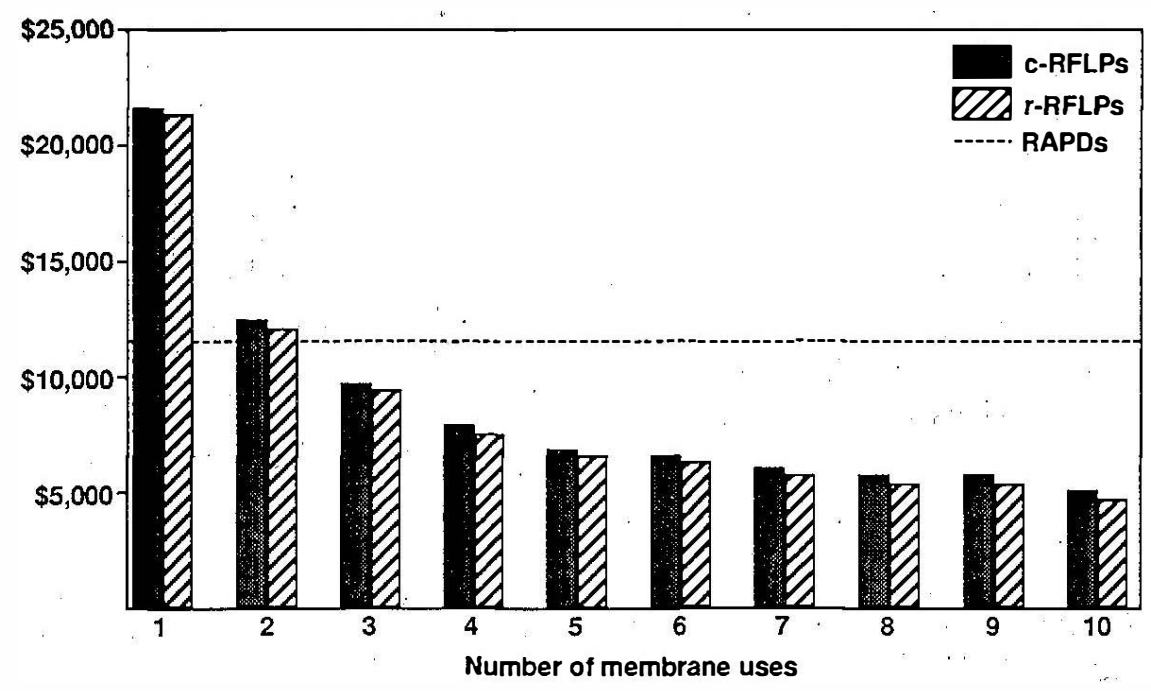

Fig. 3. Effect of membrane usage on the cost of supplies required for the genotyping of 500 individuals with 100 probes. RAPDs do not involve use of Southern blots but are shown as a reference for comparison with chemiluminescent (c-RFLPs) and radioactive (r-RFLPs) RFLP protocols 

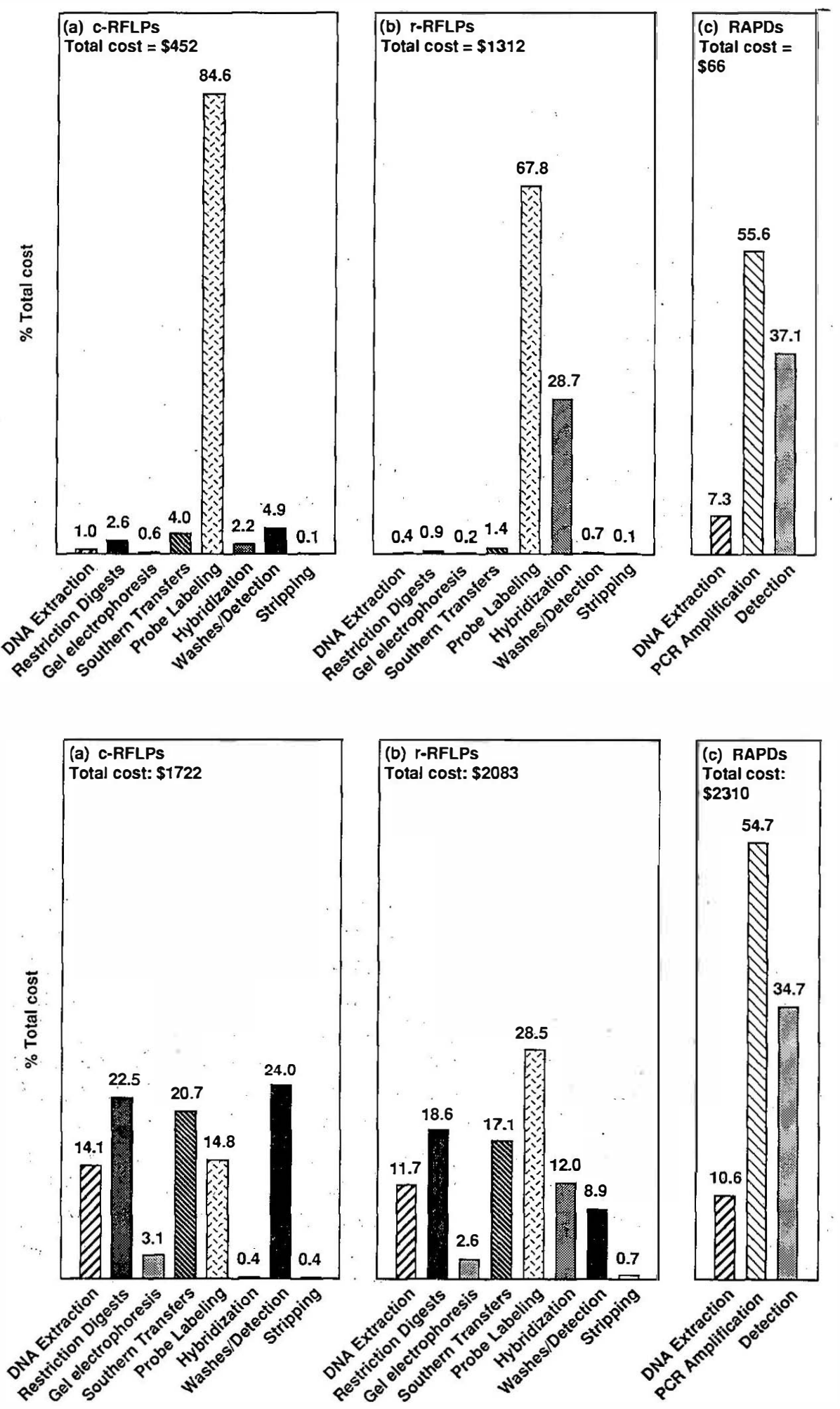

Fig. 4a-c. Allocation of costs associated with the genotyping of 100 individuals with 100 probes (primers) to the procedures of three protocols a Chemiluminescent-based RFLPs, b radioactivity-based RFLPs, and c RAPDs Figures are percentage of the total cost, indicated for each protocol in the box
Fig. 5. Allocation of costs associated with the genotyping of 2 individuals with 150 probes (primers) to the procedures of three protocols a Chemiluminescent-based RFLPs, b. radioactivity-based RFLPs, and $\mathbf{c}$ RAPDs. Figures are percentage of the total cost, indicated for each protocol in the box probes or primers). c-RFLPs were found to be the least expensive markers, r-RFLPs and RAPDs being respectively $21 \%$ and $34 \%$ more costly. A QTL mapping experiment was also simulated. Estimations of costs were divided into two parts: screening of the parents for polymorphic markers (Fig. 5a-c), and genotyping of the segregating progeny (Fig. 6a-c). Actual project costs are best estimated by adding parental screening and progeny genotyping cost. Once again, c-RFLPs were found to be the most cost efficient markers $(\$ 7,394$ for 50,000 data points). r-RFLPs and RAPDs were respectively $7 \%$ and $67 \%$ more expensive than c-RFLPs. Allocation of total costs to individual procedures revealed that PCR amplifications always accounted for more than $50 \%$ of total RAPD genotyping costs (Figs. $4 c, 5 c, 6 c)$. The enzyme Taq DNA polymerase represented over $30 \%$ of the PCR amplification costs (data not shown). 


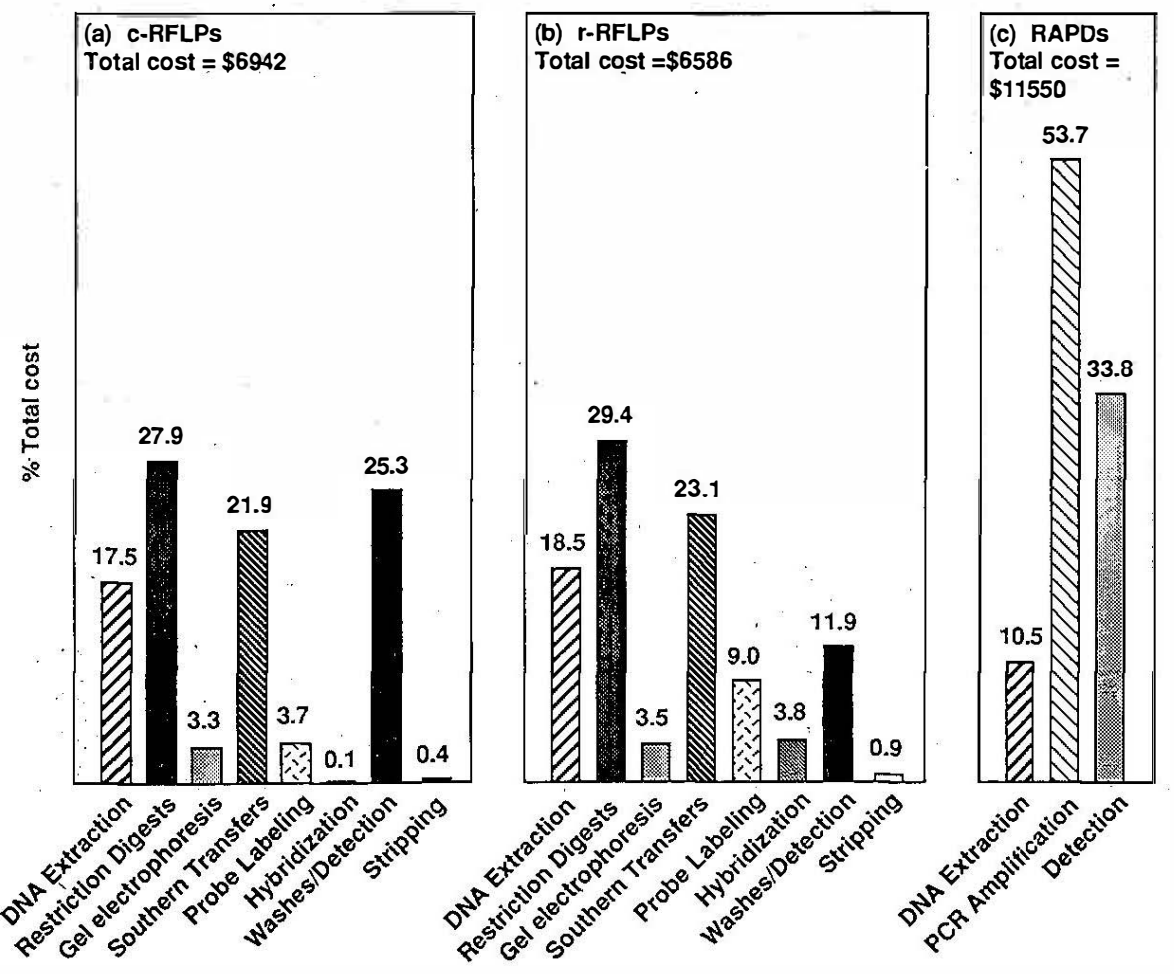

Fig. 6a-c. Allocation of costs associated with the genotyping of 500 individuals with 100 probes (primers) to the procedures of three protocols. a Chemiluminescent-based RFLPs, b radioactivity-based RFLPs, and c RAPDs. Figures are percentage of the total cost, indicated for each protocol in the box

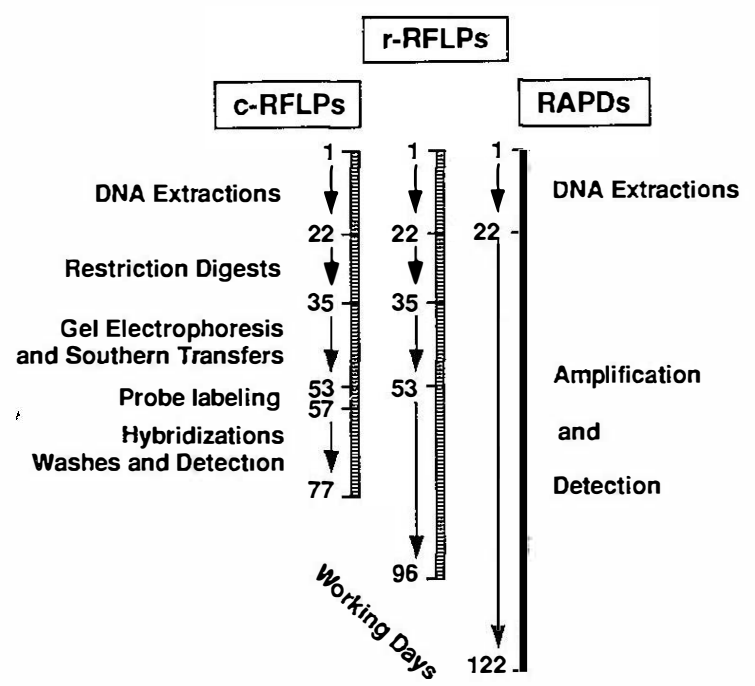

Fig. 7. 'Hands-on' time requirements for the genotyping of 500 individuals with 100 probes (primers). Figures are numbers of working days. Procedures constituting the protocols (c-RFLPs, r-RFLPs and RAPDs) are placed against their respective time scale

Estimates of the hands-on time required to, genotype 500 individuals with 100 probes or primers are shown in Fig. 7 for c-RFLPs, r-RFLPs, and RAPDs. Figures represent working days for one laboratory technician. Calculations were based on the following workload: 50 isolations of genomic DNA every other day (both for RFLPs and RAPDs); 200 restriction digests per day; ten gel electrophoreses, and ten Southern transfers every other day; labeling of 50 probes every other day; hybridization of 10 probes and washes of the corresponding membranes every other day (for
c-RFLPs) or labeling of 10 probes, hybridization and washes of corresponding membranes increasing from every other day to every week (for r-RFLPs); and 500 PCR amplifications and corresponding gel electrophoreses per day (RAPDs only). While 122 working days (976 working hours) would be required to complete the genotyping endeavor with RAPDs, c-RFLPs would require only 77 days (616 hours), or $37 \%$ less time (Fig. 7). r-RFLPs were intermediate, requiring approximately 96 days (768 hours); $21 \%$ less time than RAPDs, but $25 \%$ more time than c-RFLPs. It is very difficult to estimate the total time required for r-RFLPs, given the uncertainty of the increasing exposure times required; however, it is felt that r-RFLPs would require more time than c-RFLPs. In RFLP analyses, much time is required to make Southern blots from isolated genomic DNA. However, once membranes have been made, many data points can be generated at a very fast pace $(5,000$ data points every other day for c-RFLPs in the example shown in Fig. 7). Comparing protocols for time requirements over a range of sample sizes and number of probes or primers reveals that RAPDs are the most time efficient for generating small numbers of data points, while RFLPs are the most time efficient for generating large numbers of data points (data not shown), r-RFLPs and cRFLPs are comparable for hands-on time requirements (data not shown); however, because of the long exposure times involved, $r$-RFLPs present a very large discrepancy between hands-on and elapsed time requirements, and are therefore much less time efficient than c-RFLPs. 


\section{Discussion}

The above results clearly demonstrate that, in terms of genotyping costs and time requirements, none of the three marker protocols evaluated has absolute advantage over the others. While RAPDs are the most economical markers for relatively small- to mediumsized projects, RFLPs would be the marker of choice for more extensive genotyping endeavors. Martin et al. (1991) also suggest the use of RFLP markers in place of RAPDs for large sample sizes, thus corroborating our findings. RFLPs and RAPDs, however, provide different types and amounts of genetic information. Because maize RFLP probes were selected to represent singlecopy genomic sequences (Helentjaris et al. 1986), they generally provide information at a single marker locus. RAPD primers, on the other hand, provide information at many loci (Rafalski et al. 1991; Welsh et al. 1991), although large differences are observed across primers and species (Williams et al. 1990; Welsh et al. 1991). The benefit gained from using multiple-locus markers for fingerprinting or for the determination of relationships among genotypes would be lost when monitoring genetic changes at specific loci as in the case of backcrossing or marker-assisted selection. In certain situations, several RFLP probes can be combined in a single hybridization, thereby yielding genetic information at several marker loci simultaneously. Differences among protocols for the levels of success of laboratory work also affect the amount of information generated and ultimately the relative costs of marker classes. Walton (1990) reported a failure rate for $\mathrm{r}$ RFLPs that approached 25\%. In CIMMYT's Applied Molecular Genetics laboratory, we observed a $10-20 \%$ failure rate for both c-RFLPs and RAPDs, suggesting no significant difference in data yield among marker protocols.

Different marker classes provide genetic information not only in different amounts but also of different qualities. RFLPs are codominant markers, inherited in a simple Mendelian fashion (Botstein et al. 1980). RAPDs are usually dominant markers, thus preventing the accurate detection of heterozygotes (Williams et al. 1990; Welsh et al. 1991). Pairs of RAPD markers tightly linked in repulsion phase would allow the identification of heterozygotes (Williams et al. 1990). However, the number of markers required for such assays and the associated genotyping costs would increase accordingly. Even though dominance might not restrict the use RAPD markers (Carlson et al. 1991; Welsh et al. 1991), it affects genotyping costs and should be taken into account when designing mapping experiments (Rafalski et al. 1991) or marker-based breeding strategies.

Additional factors, although not directly related to marker characteristics, also affect the feasibility and cost of genotyping projects. Such factors include species-specific and environmental elements. Because RAPDs are based on PCR, they require much less DNA than RFLPs (Welsh et al. 1991; Williams et al. 1991) and allow the use of much simpler procedures for the isolation of genomic DNA (Williams et al. 1991), although high purity may be required as well as samples free from pathogen contamination. For maize, between 10 and $30 \mathrm{ng}$ of DNA are needed per RAPD reaction, which is about 30-200 times less than the amount needed for RFLP analysis (10 $\mu \mathrm{g}$ of DNA for five to ten hybridizations). The development of simple methods of DNA extraction may result in lower costs for RAPD genotyping. In situations in which DNA is not available in amounts large enough for RFLP analysis, RAPD genotyping is the only feasible marker approach. Megagametophytes of loblolly pine are an example of such a situation (R. Sederoff, personal communication).

To date, several hundred public maize RFLP probes are available at no charge to any researcher. As a consequence, estimations of RFLP genotyping costs in maize do not need to include costs of probe development (supplies and time). However, for a species with no RFLP history, these initial expenses must be taken into account in the calculation of RFLP genotyping costs. Doing so will affect the relative merits of RFLPs and RAPDs.

Variations in the relative costs of labor and supplies affect the relative importance of time and supplies in the assessment of genotyping costs. Such changes, in turn, affect the relative merits of marker protocols. The location of the laboratory also determines in part the availability and cost of both supplies and labor, and as a consequence the value of the labor to the supplies cost ratio. RAPD analysis may also be much more amenable to automation than RFLP-based assays, which could substantially increase the output with lower labor costs. While automation may be an important consideration under situations of high labor cost, laboratories situated in many developing countries would not be able to utilize automated protocols effectively, primarily due to high initial costs and a lack of repair expertise.

As genotyping costs are better understood, protocols should be modified with the aim of improving cost and time efficiency. Means to achieve this goal include utilization of alternative reagents, re-uses of solutions or plasticware, and the automation of laborious steps.

Becaūse genotyping costs are determined by numerous factors, some of which are very case-specific, any general statement about the relative costs of molecular marker protocols is prone to be untrue. Genotyping costs should be estimated on a project basis and used jointly with estimations of the relative benefits of 
marker classes for the choice of a molecular marker strategy.

Acknowledgments. This study was made possible in part by a fellowship from Ciba-Geigy to MR.

\section{References}

Asins MJ, Carbonell EA (1989) Distribution of genetic variability in a durum wheat world collection. Theor Appl Genet 77:287-294

Bassiri A, Adams MW (1978) Evaluation of bean cultivar relationships by means of isozyme electrophoretic patterns. Euphytica 27:707-720

Beckmann JS, Soller M (1983) Restriction fragment length polymorphisms in genetic improvement: methodologies, mapping and costs. Theor Appl Genet 67:35-43

Bernatzky R, Tanksley SD (1986) Toward a saturated linkage map in tomato based on isozymes and random cDNA sequences. Genetics 112:887-898

Botstein D, White RL, Skolnick M, Davis RW (1980) Construction of a linkage map in man using restriction fragment length polymorphisms. Am J Hum Genet 32:314-331

Burr B, Evola SV, Burr FA (1983) The application of restriction fragment length polymorphism to plant breeding. In: Setlow JK, Hollaender A (eds) Genetic engineering, principles and methods, Vol. 5. Plenum Press, New York, pp 45-59

Carlson JE, Tulsieram LK, Glaubitz JC, Luk VWK, Kauffeldt C, Rutledge R (1991) Segregation of random amplified DNA markers in $F_{1}$ progeny of conifers. Theor Appl Genet 83: 194-200

Chang C, Boowman JC, DeJohn AW, Lander ES, Meyerowitz ES (1988) Restriction fragment length polymorphism linkage map of Arabidopsis thaliana. Proc Natl Acad Sci USA $85: 6856-6860$

De Verna JW, Alpert KB (1990) RFLP technology. In: Proc Hortic Biotechnol Symp. University of California, Davis, Wiley-Liss, New York, pp 247-259

Devey ME, Jermstad KD, Tauer CG, Neale DB (1991) Inheritance of RFLP loci in a loblolly pine three-generation pedigree. Theor Appl Genet 83:238-242

Doebley JF, Goodman MM, Stuber CW (1984) Isoenzymatic variation in Zea (Gramineae). System Bot 9:203-218

Dudley JW (1989) Use of molecular markers in corn breeding. In: Proc 25th Annu Ill Corn Breed School. Urbana-Champaign, Illinois, pp 120-138

Fehr WR (1987) Principles of cultivar development, Vol. 1, theory and technique. McGraw-Hill, New York

Gebhardt C, Ritter E, Debener T, Schachtschabel U, Walkemeier B, Uhrig H, Salamini F (1989) RFLP analysis and linkage mapping in Solanum tuberosum. Theor Appl Genet 78:65-75

Gebhardt C, Ritter E, Barone A, Debener T, Walkemeier B, Schachtschabel U, Kaufmann H, Thompson RD, Bonierbale MW, Ganal MW, Tanksley SD, Salamini F (1991) RFLP maps of potato and their alignment with the homeologous tomato genome. Theor Appl Genet 83:49-57

Goodman MM, Stuber CW (1980) Genetic identification of lines and crosses using isoenzyme electrophoresis. In: Proc 35th Annu Corn Sorghum Industry Res Conf American Seed Trade Assoc, Vol. 35, pp 10-31

Grodzicker T, Williams J, Sharp P, Sambrook J (1974) Physical mapping of temperature-sensitive mutations of adenoviruses. Cold Spring Harbor Symp Quant Biol 39:439-446
Garner A, Jahoor A, Schondelmaier J, Siedler H, Pillen K, Fischbeck G, Wenzel G, Herrmann RG (1991) Construction of an RFLP map of barley. Theor Appl Genet 83:250-256

Helentjaris T, Slocum M, Wright S, Schaefer A, Nienhuis J (1986) Construction of genetic linkage maps in maize and tomato using restriction fragment length polymorphisms. Theor Appl Genet 72:761-769

Heun M, Kennedy AE, Anderson JA, Lapitan NLV, Sorrels ME, Tanksley SD (1991) Construction of a restriction fragment length polymorphism map for barley (Hordeum vulgare). Genome 34:437-447

Hoisington DA (1992) Laboratory protocols. CIMMYT Applied Molecular Genetics Laboratory, Mexico, D.F., CIMMYT

Horsington DA, Coe EH (1990) Mapping in maize using RFLPs. In: Gustafson JP (ed) Gene manipulation in plant improvement II. Plenum Press, New York, pp 331-352

Keim P, Diers BW, Olson TC, Shoemaker RC (1990) RFLP mapping in soybean: association between marker loci and variation in Quantitative traits. Genetics 126:735-742

Kesseli RV, Michelmore RW (1986) Genetic analysis and phylogenies detected from isozyme markers among species of Lactuca. J Hered 77:324-331

Landry BS, Kesseli RV, Farrara B, Michelmore RW (1987) A genetic map of lettuce (Lactuca sativa L.) with restriction fragment length polymorphisms, isozyme, disease resistance and morphological markers. Genetics 116:331-337

Martin GB, Williams JGK, Tanksley SD (1991) Rapid identification of markers linked to a Pseudomonas resistance gene in tomato by using random primers and near-isogenic lines. Proc Natl Acad Sci USA 88:2336-2340

McCoucgh SR, Kochert G, Yu ZH, Wang ZY, Khush GS, Coffman WR, Tanksley SD (1988) Molecular mapping of rice chromosomes. Theor Appl Genet 76:815-829

McIntyre CL (1988) Variation at isozyme loci in Triticeae. Plant Syst Evol 160:123-142

Murray MG, Romero-Severson J, West DP, Cramer JH (1988) Restriction fragment length polymorphisms: what are they and how can breeders use them? In: Proc 43rd Annual Corn Sorghum Industry Res Conf. American Seed Trade Assoc, Vol. 43, pp 72-87

Nam H-G, Giraudat J, den Boer B, Moonan F, Loos WDB, Hauge BM, Goodman HM (1989) Restriction fragment length polymorphism linkage map of Arabidopsis thaliana. Plant Cell 1:699-705

Osborn TC, Alexander DC, Fobes JF (1987) Identification of restriction fragment length polymorphisms linked to genes controlling soluble solids content in tomato fruit. Theor Appl Genet 73:350-356

Rafalski JA, Tingey SV, Williams JGK (1991) RAPD markers a new technology for genetic mapping and plant breeding. AgBiotech News Info 3:645-648

Sax K (1923) The association of size differences with seed-coat pattern and pigmentation in Phaseolus vulgaris. Genetics $8: 552-560$

Shin JS, Chao S, Corpuz L, Blake T (1990) A partial map of the barley genome incorporating restriction fragment length polymorphism, polymerase chain reaction, isozyme and morphological marker loci. Genome 33: 803-810

Smith JSC, Smith OS (1989) The use of morphological, biochemical, and genetic characteristics to measure distance and to test for minimum distance between inbred lines of maize (Zea mays L.). (Paper presented at UPOV workshop, Versailles, France). Pioneer Hi-Bred Int, Johnston, Iowa

Smith JSC, Goodman MM, Stuber CW (1985a) Genetic variability within US maize germplasm. I. Historically important lines. Crop Sci 25:550-555 
Smith JSC, Goodman MM, Stuber CW (1985b) Genetic variability withın US maize germplasm. II. Widely-used inbred lines 1970 to 1979. Crop Sci 25:681-685

Smith JSC, Smith OS, Wall SJ (1991) Associations among widely used French and US maize hybrids as revealed by restriction fragment length polymorphisms. Euphytica 54:263-273

Soller M, Beckmann JS (1983) Genetıc pólymorphism in varietal identification and genetic improvement. Theor Appl Genet $67: 25-33$

Song KM, Suzuki JY, Slocum MK, Williams PH, Osborn TC (1991) A linkage map of Brassica rapa (syn. campestris) based on restriction fragment length polymorphism loc1. Theor Appl Genet 82:296-304

Stuber CW, Edwards MD (1986) Genotypic selection for improvement of quantitative traits in corn using molecular marker loci. In: Proc 41st Annu Corn Sorghum Industry Res Conf. American Seed Trade Assoc, Vol. 41, pp 70-83

Tanksley SD, Young ND, Paterson AH, Bonierbale MW (1989) RFLP mapping in plant breeding: new tools for an old science. Bio/Technology 7:257-264
Walton M (1990) Application of RFLP technology to applied plant breeding. In: Proc Hortic Biotechnol Symp. University of Californıa, Davis, Wiley-Liss, New York, pp 335-346

Weining S, Langridge P (1991) Identification and mapping of polymorphisms in cereals based on the polymerase chain reaction. Theor Appl Genet 82:209-216

Welsh J, McClelland M (1990) Fingerprinting genomes using PCR with arbitrary primers. Nucleic Acids Res 18:7213-7218

Welsh J, Honeycutt RJ, McClelland M, Sobral BWS (1991) Parentage determination in maize hybrids using the arbitrarily primed polymerase chain reaction (AP-PCR). Theor Appl Genet 82:473-476

Williams JGK, Kubelik AR, Livak KJ, Rafalski A, Tingey SV (1990) DNA polymorphisms amplified by arbitrary primers are useful as genetic markers. Nucleic Acids Res 18:65136535

Williams MNV, Pande N, Nair S, Mohan M, Bennett J (1991) Restriction fragment length polymorphism analysis of polymerase chain reaction products amplified from mapped loci of rice (Oryza sativa L.) genomic DNA. Theor Appl Genet 82:489-498 